\title{
KINEMATICS OF DISK PLANETARY NEBULAE
}

\author{
W.J. MACIEL and C.M. DUTRA \\ Instituto Astronômico e Geofísico da USP, Av. Miguel Stefano 4200, 04301-904 São Paulo SP, \\ Brazil
}

In the past few years, it has become clear that planetary nebulae (PN) are a true stage in stellar evolution, especially regarding their chemical composition. This fact led to the introduction of the classification scheme developed by Peimbert, which has been applied to a sample of galactic objects for which a detailed amount of data exists.

On the other hand, the kinematical properties of PN make them interesting objects to study the kinematics of the disk population. In particular, there are controversies regarding the rotation curve of the Galaxy outside the solar circle, and it is not clear whether the curve decreases, remains constant or even increases thereafter. Since PN are luminous objects that can be observed to relatively large. distances and have accurately measured velocities, it is interesting to investigate their possible contribution to this problem.

In the present work, we will explore the kinematical properties of the different types of $\mathrm{PN}$ in comparison with classical population I objects. We will concentrate on the space distribution and kinematics of a sample containing 150 disk nebulae, especially considering the determination of the galactic rotation curve and of Oort's constants.

It is shown that planetary nebulae of Types I, IIa, IIb, III, and IV form an approximately continuous sequence in terms of these properties, confirming similar conclusions based on their chemical composition. Some kinematical consequences regarding the connection between planetary nebulae and HII regions are explored, leading to the determination of the galactic rotation curve and Oort's constants. It is found that the rotation curve presents a flattening near the solar circle and a moderate increase for larger galactocentric distances.

Work partially supported by CNPq and FAPESP. 